\title{
Accuracy of direct genomic values derived from imputed single nucleotide polymorphism genotypes in Jersey cattle
}

\author{
K. A. Weigel, ${ }^{* 1}$ G. de los Campos, ${ }^{\star}$ A. I. Vazquez, ${ }^{*}$ G. J. M. Rosa, ${ }^{*} \dagger$ D. Gianola, ${ }^{\star} † \ddagger$ and C. P. Van Tassell§\# \\ *Department of Dairy Science, \\ †Department of Biostatistics and Medical Informatics, and \\ ‡Department of Animal Sciences, University of Wisconsin, Madison 53706 \\ $\S B$ Bovine Functional Genomics Laboratory, and \\ \#Animal Improvement Programs Laboratory, ARS, USDA, Beltsville, MD 20705-2350
}

ABSTRACT

The objective of the present study was to evaluate the predictive ability of direct genomic values for economically important dairy traits when genotypes at some single nucleotide polymorphism (SNP) loci were imputed rather than measured directly. Genotypic data consisted of 42,552 SNP genotypes for each of 1,762 Jersey sires. Phenotypic data consisted of predicted transmitting abilities (PTA) for milk yield, protein percentage, and daughter pregnancy rate from May 2006 for 1,446 sires in the training set and from April 2009 for 316 sires in the testing set. The SNP effects were estimated using the Bayesian least absolute selection and shrinkage operator (LASSO) method with data of sires in the training set, and direct genomic values (DGV) for sires in the testing set were computed by multiplying these estimates by corresponding genotype dosages for sires in the testing set. The mean correlation across traits between DGV (before progeny testing) and PTA (after progeny testing) for sires in the testing set was $70.6 \%$ when all 42,552 SNP genotypes were used. When genotypes for 93.1, 96.6, 98.3 , or $99.1 \%$ of loci were masked and subsequently imputed in the testing set, mean correlations across traits between DGV and PTA were 68.5, 64.8, 54.8, or $43.5 \%$, respectively. When genotypes were also masked and imputed for a random $50 \%$ of sires in the training set, mean correlations across traits between DGV and PTA were $65.7,63.2,53.9$, or $49.5 \%$, respectively. Results of this study indicate that if a suitable reference population with high-density genotypes is available, a low-density chip comprising 3,000 equally spaced SNP may provide approximately $95 \%$ of the predictive ability observed with the BovineSNP50 Beadchip (Illumina Inc., San Diego, CA) in Jersey cattle. However, if fewer

Received February 8, 2010.

Accepted July 5, 2010.

${ }^{1}$ Corresponding author: kweigel@wisc.edu than 1,500 SNP are genotyped, the accuracy of DGV may be limited by errors in the imputed genotypes of selection candidates.

Key words: genomic selection, imputation, Jersey, predictive ability

\section{INTRODUCTION}

The recent introduction of high-throughput assays for dense genotyping of SNP in cattle (Van Tassell et al., 2008) has stimulated hundreds of research projects and transformed practical breeding programs. Tens of thousands of dairy cattle, mostly progeny-tested bulls in commercial AI programs or young bulls that are candidates for such programs, have been genotyped using the BovineSNP50 BeadChip (Illumina Inc., San Diego, $\mathrm{CA}$ ) during the past $2 \mathrm{yr}$. Numerous methods have been proposed for estimating SNP effects and predicting direct genomic values (DGV) of selection candidates (e.g., Hayes et al., 2009), some of which are variations of the whole-genome selection model proposed in the ground-breaking publication of Meuwissen et al. (2001). In North America, genomic information has been used in national genetic evaluations for routine calculation of PTA for production, conformation, and fitness of dairy cattle since January 2009 (Wiggans et al., 2009).

Despite the impressive gains in reliability of young selection candidates that have been achieved using current high-density genotyping platforms, such as the BovineSNP50 BeadChip (VanRaden et al., 2009), the commercial price of such assays may limit their application to males and elite females. Inexpensive, lowdensity genotyping platforms with, for example, 300 to 3,000 SNP, could stimulate widespread commercial use for applications such as preliminary screening of young bulls, selection of replacement heifers, discovery of parentage, identification of optimal mating sires, or development of genome-guided management protocols. In practice, the optimal size of such platforms and the characteristics of the SNP that comprise them will depend on the population and family structure, the 
extent of linkage disequilibrium (LD) in the species and breed of interest, the genetic architecture underlying economically important traits, the number and proportion of animals with high-density SNP genotypes and accurate phenotypes (i.e., the training set), and the extent of relationships between these individuals and future candidates for selection (i.e., the testing set).

In a recent study, Weigel et al. (2009) found that genotyping young Holstein bulls for 300 to 2,000 highly selected SNP could provide DGV for lifetime net merit with correlations of 0.43 to 0.57 with future PTA from progeny testing, compared with a correlation of 0.61 when all markers on the BovineSNP50 BeadChip were used. Furthermore, Vazquez et al. (2009) reported that low-density genotyping platforms with 500 to 1,000 selected SNP, where selection was based on the largest estimated SNP effects for individual production or fitness traits, could provide correlations of 0.55 to 0.65 with subsequent PTA from progeny testing in Holstein cattle. Furthermore, Vazquez et al. (2009) noted that a low-density platform composed of SNP with the largest effects for lifetime net merit in Holsteins provided correlations of 0.40 to 0.55 with progeny-test PTA for specific production and fitness traits, but correlations tended to be higher for production than for fitness. These challenges, coupled with technological advances that continue to drive down the per locus cost of genotyping, suggest that one should consider genotyping a slightly larger set of equally spaced SNP that would facilitate imputation of high-density SNP genotypes using haplotypes from a reference population that has already been genotyped on a high-density platform.

Numerous algorithms and public domain software packages have been developed for construction of haplotypes and imputation of genotypes in humans (e.g., Scheet and Stephens, 2006; Kong et al., 2008; Howie et al., 2009). In most applications involving humans, the objective is to combine data sets containing subjects (usually cases and controls) who have been genotyped with different high-density platforms for the purpose of carrying out a genome-wide association study for one or more disease traits (e.g., Halperin and Stephan, 2009; Hao et al., 2009). Combining data from competing high-density SNP arrays has not yet been a major issue in cattle, because the majority of genotyping has involved the Illumina BovineSNP50 BeadChip. However, it is likely that multiple high-density (e.g., tens of thousands of SNP) and ultra-high-density (e.g., hundreds of thousands of SNP) genotyping platforms will soon be commercially available, and combining data from different platforms may become relevant in cattle as well.

In food animal species, an alternative use of haplotyping and imputation could involve genotyping a large number of potential selection candidates using an inexpensive, low-density genotyping platform containing equally spaced SNP, and subsequently using this information in conjunction with high-density SNP genotypes (and perhaps pedigrees) of animals in a reference panel to impute missing high-density genotypes in the selection candidates. In a simulation study, Habier et al. (2009) noted that low-density genotyping of selection candidates in the current generation with equally spaced SNP, coupled with high-density genotyping of their parents and grandparents, could lead to rapid and cost-effective genetic progress in commercial breeding programs, particularly if the newly selected parents in each generation were re-genotyped on the high-density platform. Recently, Weigel et al. (2010) masked varying proportions of BovineSNP50 genotypes on 3 chromosomes in Jersey cattle and showed that publicly available, population-based algorithms could impute more than $90 \%$ of masked genotypes correctly when as few as 2,000 to 3,000 loci were unmasked in the study sample. However, the effect of imputation errors on the predictive ability of resulting DGV has not yet been documented.

The objective of the present study was to evaluate the predictive ability of DGV for economically important dairy traits when a large proportion of SNP genotypes have been imputed rather than measured directly. Specifically, we sought to determine the reduction in accuracy of DGV for milk yield, milk composition, and female fertility that should be expected if the majority of young selection candidates, and perhaps also a significant proportion of their ancestors, were genotyped using low-density panels consisting of varying numbers of equally spaced SNP.

\section{MATERIALS AND METHODS}

Genotypes of 1,762 Jersey sires were provided by the USDA-ARS Animal Improvement Programs Laboratory (Beltsville, MD) and consisted of 42,552 SNP markers distributed across the 29 Bos taurus autosomes and the $\mathrm{X}$ chromosome. These represented the subset of SNP on the BovineSNP50 BeadChip that are used for routine genomic evaluation of dairy cattle in the United States (Wiggans et al., 2009) and were obtained after removal of SNP with (1) a call rate of less than 90\%; (2) greater than $1 \%$ parent-progeny conflicts; (3) complete LD with an adjacent SNP; (4) minor allele frequency of less than $1 \%$ in each of the Holstein, Jersey, and Brown Swiss breeds, or (5) unknown physical position on the chromosome based on the UMD2 assembly of B. taurus (Zimin et al., 2009). Genotypes at each locus were coded as 0 (homozygous for allele B), 1 (heterozygous), 2 (homozygous for allele A), or missing. 
Table 1. Summary of data from the US Jersey cattle population used in the present study

\begin{tabular}{|c|c|c|}
\hline Item & $\begin{array}{c}\text { Training set } \\
(\mathrm{n}=1,446 \text { bulls })\end{array}$ & $\begin{array}{c}\text { Testing set } \\
(\mathrm{n}=316 \text { bulls })\end{array}$ \\
\hline \multicolumn{3}{|l|}{ Year of birth } \\
\hline 1950-1954 & 1 & 0 \\
\hline 1955-1959 & 3 & 0 \\
\hline 1960-1964 & 5 & 0 \\
\hline 1965-1969 & 12 & 0 \\
\hline 1970-1974 & 37 & 0 \\
\hline 1975-1979 & 35 & 0 \\
\hline 1980-1984 & 82 & 0 \\
\hline $1985-1989$ & 199 & 0 \\
\hline 1990-1994 & 375 & 0 \\
\hline 1995-1999 & 509 & 6 \\
\hline $2000-2004$ & 188 & 310 \\
\hline \multicolumn{3}{|c|}{ Ancestors in the training set } \\
\hline Sire & 1,233 & 287 \\
\hline Maternal grandsire & 1,196 & 280 \\
\hline PTA & (May 2006) & (April 2009) \\
\hline \multicolumn{3}{|l|}{ Milk yield } \\
\hline No. of daughters & $660 \pm 69$ & $84 \pm 8$ \\
\hline Reliability, \% & $87.8 \pm 0.2$ & $83.9 \pm 0.4$ \\
\hline \multicolumn{3}{|l|}{ Protein percentage } \\
\hline No. of daughters & $631 \pm 69$ & $84 \pm 8$ \\
\hline Reliability, \% & $87.8 \pm 0.2$ & $83.9 \pm 0.4$ \\
\hline \multicolumn{3}{|c|}{ Daughter pregnancy rate } \\
\hline No. of daughters & $380 \pm 34$ & $73 \pm 7$ \\
\hline Reliability, \% & $64.0 \pm 0.4$ & $53.7 \pm 0.5$ \\
\hline
\end{tabular}

Phenotypes, which were also provided by the USDAARS Animal Improvement Programs Laboratory, represented the results of progeny testing and consisted of PTA for milk yield, protein percentage, and daughter pregnancy rate (DPR); each trait was standardized to a sample variance of 1 . As shown in Table 1 , the training set comprised 1,446 sires with official PTA (i.e., at least 10 daughters) for milk yield, protein percentage, and DPR in May 2006, whereas the testing set comprised 316 sires that obtained their first official PTA between May 2006 and April 2009. Note that the April 2009 PTA used in the present study were traditional animal-model BLUP evaluations based only on phenotypic data, rather than officially published PTA that included both phenotypic and genomic information. As shown in Table 1, the majority of sires and maternal grandsires of animals in the training and testing sets had been genotyped and were therefore present in the training set. In fact, almost all North American Jersey sires that have been marketed through commercial AI programs have been genotyped with the BovineSNP50 Beadchip. The average reliabilities for milk yield, protein percentage, and DPR for sires in the testing set in April 2009 were 83.9, 83.9, and 53.7\%, respectively, so the realized accuracy of genomic predictions computed in the present study may have been reduced slightly by measurement errors in the quantities we attempted to predict, especially for DPR.
As noted previously, genotypes for 42,552 SNP were available for all sires that were represented in the training and testing sets. Our objective was to evaluate the effect of genotype imputation on the accuracy of DGV, or more specifically the effect of imputation errors that might arise when low-density genotypes from inexpensive chips are used to impute high-density genotypes for the purpose of whole genome selection. We began with a subset of 3,000 SNP from the BovineSNP50 Beadchip that were selected by the USDA-ARS Bovine Functional Genomics Laboratory (Beltsville, MD) as candidates for a low-density chip based on equidistant physical location and high minor allele frequency across a broad range of Bos taurus and Bos indicus breeds. After applying the edits described previously, a total of 2,942 potential low-density SNP remained for analysis.

Two alternative genotyping strategies were considered. In both cases we masked genotypes for $93.1 \%$ (all but 2,942), 96.6\% (all but 1,468), 98.3\% (all but 741), or $99.1 \%$ (all but 366 ) of the 42,552 original SNP loci. The latter 3 subsets of SNP were obtained by ordering the original list of 2,942 SNP by chromosome and physical location within chromosome and then picking every second, fourth, or eighth SNP. A breakdown of the number of SNP per chromosome, in total and for each alternative masking rate, is given in Table 2 . The number of unmasked SNP per chromosome ranged from 48 to 175 for a masking rate of $93.1 \%, 24$ to 89 
Table 2. Number of unmasked SNP genotypes per chromosome in each of the masking rate scenarios considered in the present study

\begin{tabular}{|c|c|c|c|c|c|}
\hline \multirow[b]{2}{*}{ Chromosome } & \multicolumn{5}{|c|}{ Masking rate, $\%$} \\
\hline & 0.0 & 93.1 & 96.6 & 98.3 & 99.1 \\
\hline BTA1 & 2,693 & 175 & 89 & 44 & 20 \\
\hline BTA2 & 2,242 & 152 & 75 & 39 & 20 \\
\hline BTA3 & 2,072 & 132 & 66 & 32 & 17 \\
\hline BTA4 & 2,005 & 134 & 67 & 34 & 17 \\
\hline BTA5 & 1,770 & 136 & 67 & 34 & 17 \\
\hline BTA6 & 2,016 & 133 & 66 & 34 & 17 \\
\hline BTA7 & 1,812 & 125 & 62 & 32 & 15 \\
\hline BTA8 & 1,917 & 124 & 62 & 31 & 16 \\
\hline BTA9 & 1,662 & 119 & 60 & 29 & 15 \\
\hline BTA10 & 1,750 & 117 & 58 & 30 & 14 \\
\hline BTA11 & 1,809 & 120 & 59 & 30 & 16 \\
\hline BTA12 & 1,389 & 101 & 51 & 26 & 11 \\
\hline BTA13 & 1,451 & 93 & 47 & 24 & 11 \\
\hline BTA14 & 1,471 & 95 & 48 & 23 & 12 \\
\hline BTA15 & 1,376 & 93 & 46 & 23 & 12 \\
\hline BTA16 & 1,330 & 89 & 44 & 23 & 11 \\
\hline BTA17 & 1,322 & 83 & 41 & 21 & 10 \\
\hline BTA18 & 1,094 & 71 & 36 & 18 & 8 \\
\hline BTA19 & 1,125 & 71 & 35 & 18 & 9 \\
\hline BTA20 & 1,272 & 80 & 41 & 20 & 10 \\
\hline BTA21 & 1,132 & 79 & 40 & 20 & 10 \\
\hline BTA22 & 1,066 & 66 & 33 & 17 & 7 \\
\hline BTA23 & 902 & 59 & 29 & 15 & 8 \\
\hline BTA24 & 1,014 & 70 & 35 & 17 & 9 \\
\hline BTA25 & 812 & 48 & 24 & 12 & 6 \\
\hline BTA26 & 885 & 57 & 28 & 15 & 7 \\
\hline BTA27 & 812 & 51 & 25 & 13 & 6 \\
\hline BTA28 & 795 & 51 & 25 & 13 & 7 \\
\hline BTA29 & 879 & 57 & 28 & 14 & 7 \\
\hline $\mathrm{X}$ & 677 & 161 & 81 & 40 & 21 \\
\hline Total & 42,552 & 2,942 & 1,468 & 741 & 366 \\
\hline
\end{tabular}

for a masking rate of $96.6 \%, 12$ to 44 for a masking rate of $98.3 \%$, and 6 to 20 for a masking rate of $99.1 \%$. In the first genotyping strategy, we masked genotypes for all sires that were in the testing set, whereas in the second scenario we masked genotypes for all sires that were in the testing set plus a randomly chosen $50 \%$ of sires from the training set. The first genotyping strategy sought to mimic a situation in which all actively marketed AI sires were genotyped using a high-density platform and all young candidates for selection were genotyped using an inexpensive, low-density platform. The second genotyping strategy sought to mimic a situation in which a significant proportion of the actively marketed AI sires (including many bulls that would be used to estimate SNP effects) were also genotyped only on a low-density platform. One might expect to encounter the former situation during the first year or two after low-density genotyping platforms become commercially available, whereas one might encounter the latter situation after such platforms have been used widely for several years.

Next, masked genotypes were imputed using the algorithm of Howie et al. (2009), which was implemented via the IMPUTE 2.0 software (Department of Sta- tistics, University of Oxford, United Kingdom). This algorithm accommodates multiple reference panels of phased or unphased genotypes that may represent different SNP sets of varying density. The algorithm estimates haplotypes at SNP that are present in both the reference panel (i.e., training set) and the study sample (i.e., testing set) and then imputes genotypes in the latter, assuming that these haplotype guesses are correct. Uncertainty about phasing is taken into account by iterating these steps in a Markov chain Monte Carlo framework. Thus, IMPUTE 2.0 gains accuracy by using information from the reference panels and the study sample during phasing. To enhance computational feasibility, it uses a subset of haplotypes when building the conditional distribution of haplotypes for an animal in the study sample in a given iteration, considering the animal's genotype, the haplotypes of other animals in the study sample, and the haplotypes of animals in the reference panel. These "conditioning states" correspond to selected sets of haplotypes that are "closest" to the animal in question. For computational reasons, the number of conditioning states was set to 40 in the present study, as in the aforementioned study of Weigel et al. (2010). Howie et al. (2009) noted that slight in- 
creases in accuracy might be achieved by increasing the number of conditioning states, albeit with a quadratic increase in computing time. In large data sets, the computational feasibility of programs such as IMPUTE 2.0 can be enhanced by breaking chromosomes into pieces of more manageable size before analysis. In the present study, BTA1 to BTA11 were broken into 3 pieces of equal size, BTA12 to BTA24 were broken into 2 pieces, and BTA25 to X were analyzed in their entirety. Note that in the first genotyping strategy, in which genotypes were masked only for sires in the testing set, 1,446 sires in the training set provided reference haplotypes for imputation of high-density genotypes from the lowdensity genotypes of 316 sires in the testing set. In the second genotyping strategy, 723 sires that represented a randomly chosen $50 \%$ of animals in the training set provided reference haplotypes for imputation of highdensity genotypes in the remaining 1,039 sires (316 from the testing set and 723 from the training set).

Because uncertainty exists in the imputed genotypes, it is possible to apply a minimum threshold when calling genotypes and, in turn, to leave genotypes missing for loci at which the probability of the most likely genotype fails to meet this user-defined threshold. An alternative, which was the approach utilized in the present study, is to use the genotype probabilities provided by IMPUTE 2.0 (which range from 0 to 1 for each of the 3 possible genotypes of 0,1 , and 2 at a given locus) to compute the expected "dosage" value at each locus for each individual. The dosage value simply represents the sum of genotypes $(0,1$, and 2$)$ multiplied by their respective probabilities ( 0.00 to 1.00$)$, which is a continuous covariate that ranges from 0.00 to 2.00 for each locus, rather than a set of 3 discrete values.

After imputation of masked genotypes, the May 2006 PTA for milk yield, protein percentage, and DPR of sires in the training set were regressed on marker covariates using the Bayesian least absolute selection and shrinkage operator (LASSO) method of Park and Casella (2008). This method has been used previously in the context of genomic selection (e.g., de los Campos et al., 2009; Weigel et al., 2009). Briefly, the probability model of the Bayesian LASSO is defined as follows:

Likelihood: $p\left(\mathbf{y} \mid \mu, \boldsymbol{\beta}, \sigma_{\varepsilon}^{2}\right)=\prod_{i=1}^{n} N\left(y_{i} \mid \mu+\sum_{j=1}^{p} x_{i j} \beta_{j}, \sigma_{\varepsilon}^{2}\right)$,

Prior: $p\left(\mu, \boldsymbol{\beta}, \sigma_{\varepsilon}^{2}, \boldsymbol{\tau}^{2} \lambda,\right) \propto\left\{\prod_{j=1}^{p} N\left(\beta_{j} \mid 0, \sigma_{\varepsilon}^{2} \tau_{j}^{2}\right)\right\} \chi^{-2}\left(\sigma_{\varepsilon}^{2} \mid d f_{\varepsilon}, S_{\varepsilon}\right)$ $\left\{\prod_{j=1}^{p} \operatorname{Exp}\left(\tau_{j}^{2} \mid \lambda^{2}\right)\right\} G\left(\lambda^{2} \mid \alpha_{1}, \alpha_{2}\right)$ where $\mathbf{y}=\left(y_{1}, \ldots, y_{n}\right)^{\prime}$ is the response vector; $\mu$ is an effect common to all subjects; $\boldsymbol{\beta}=\left(\beta_{1}, \ldots, \beta_{p}\right)^{\prime}$ is a vector of marker effects; $\sigma_{\varepsilon}^{2}$ is a residual variance; $N\left(y_{i} \mid \mu+\sum_{j=1}^{p} x_{i j} \beta_{j}, \sigma_{\varepsilon}^{2}\right)$ is a normal density centered at $\mu+\sum_{j=1}^{p} x_{i j} \beta_{j}$ and with variance $\sigma_{\varepsilon}^{2} ; \boldsymbol{\tau}=\left(\tau_{1}, \ldots, \tau_{p}\right)^{\prime}$ is a vector of scale parameters controlling the prior densities of marker effects, $N\left(\beta_{j} \mid 0, \sigma_{\varepsilon}^{2} \tau_{j}^{2}\right)$, which are normal centered at zero and with variance $\sigma_{\varepsilon}^{2} \tau_{j}^{2} ; \chi^{-2}\left(\sigma_{\varepsilon}^{2} \mid d f_{\varepsilon}, S_{\varepsilon}\right)$ is a scaled inverted chi-squared distribution with degrees of freedom $d f_{\varepsilon}$ and inverted scale parameter $S_{\varepsilon}$; $\operatorname{Exp}\left(\tau_{j}^{2} \mid \lambda^{2}\right)$ is an exponential distribution assigned to a positive scale parameter $\tau_{j}^{2} ; \lambda$ is a regularization parameter for the shape of the prior distribution assigned to the $\tau_{j}^{2}$; and $G\left(\lambda^{2} \mid \alpha_{1}, \alpha_{2}\right)$ is a gamma distribution with shape and rate parameters $\alpha_{1}$ and $\alpha_{2}$, respectively. All fully conditional distributions have closed form and a Gibbs sampler can be used to draw samples from the posterior distribution. All of the Bayesian LASSO analyses were implemented via the Gibb's sampler made available by de los Campos et al. (2009), which is implemented in the $\mathrm{R}$ language ( $\mathrm{R}$ Foundation for Statistical Computing, 2009). The following specifications were assumed for prior distributions: $d f_{\varepsilon}=4, S_{\varepsilon}=1, \alpha_{1}=5$, and $\alpha_{2}=5 \times 10^{-4}$.These values give priors that yield high mass over a wide range of relevant values of the corresponding parameters. A chain of 120,000 samples was run, and the first 10,000 samples were discarded as burn-in. Posterior summaries were computed using a thinning rate of 50 . Convergence was checked by visual inspection of trace plots.

First, the Bayesian LASSO model was fitted to training data consisting of May 2006 PTA for milk yield, protein percentage, and DPR for 1,446 Jersey sires using all of the original 42,552 SNP genotypes as covariates. This yields estimates of effects, $\left\{\hat{\mu}, \hat{\beta}_{j}\right\}$, for each of the traits. These estimates can then be used to compute DGV for each trait and animal in the testing set as $\hat{y}_{i}=\hat{\mu}+\sum_{j=1}^{p} x_{i j} \hat{\beta}_{j}$. The correlation between these DGV and April 2009 animal model PTA from progeny testing (which contained no genomic information) were used to assess predictive ability. The resulting correlations are denoted as reference values in Figures 2, 3 and 4. 
Next, DGV were computed using the imputed genotypes of sires in the testing set. The estimated effects from the "full" Bayesian LASSO model described above, $\left\{\hat{\mu}, \hat{\beta}_{j}\right\}$, were used to compute DGV using imputed genotypes as

$$
\tilde{\hat{y}}_{i}=\hat{\mu}+\sum_{j \in\left\{\begin{array}{c}
\text { Observed } \\
\text { Set }
\end{array}\right\}} x_{i j} \hat{\beta}_{j}+\sum_{j \in\left\{\begin{array}{r}
\text { Masked } \\
\text { Set }
\end{array}\right\}} \tilde{x}_{i j} \hat{\beta}_{j},
$$

where $\tilde{x}_{i j}$ is an imputed dosage value. This was done for each of the 4 masking rates considered in this study. For example, with a masking rate of $93.1 \%$, the 42,552 genotype dosage values for sires in the testing set consisted of 2,942 actual genotypes and 39,610 imputed dosage values (i.e., genotype probabilities multiplied by 0,1 , and 2). For masking rates of 96.6, 98.3, and 99.1\%, the corresponding numbers of actual genotypes for sires in the testing set were $1,468,741$, and 366 , respectively, whereas the corresponding numbers of imputed dosage values were $41,084,41,811$, and 42,186 , respectively.

Because a key question regarding low-density platforms is the difference in predictive ability that one should expect when imputing missing high-density genotypes versus the alternative of removing loci with a large proportion of missing genotypes from the analysis, we also fit 4 "reduced" Bayesian LASSO models to data of sires in the training set. These models contained 2,942, 1,468, 741, or 366 markers, respectively, for masking rates of 93.1,96.6,98.3, or $99.1 \%$, and loci selected for these reduced models corresponded to SNP that were unmasked in the respective testing sets. As before, estimated regression coefficients were multiplied by observed SNP genotypes and summed across markers, for each of the $2,942,1,468,741$, or 366 unmasked loci to obtain DGV, and these were subsequently correlated with April 2009 PTA from progeny testing.

Finally, an important issue with respect to the design of breeding programs based on low-density genotyping is the potential loss in accuracy that might occur in future generations if some animals whose phenotypes contribute to the estimation of SNP effects (i.e., members of the training set) lack high-density genotypes. For this reason, we employed a second genotyping strategy in which $50 \%$ of the sires in the training set (chosen at random) also had only low-density SNP genotypes. In this case, the 42,552 high-density genotypes used for estimation of SNP effects in the full Bayesian LASSO model represented a mixture of 723 Jersey sires with 42,552 actual genotypes and 723 Jersey sires with $2,942,1,468,741$, or 366 actual genotypes and $39,610,41,084,41,811$, or 42,186 dosage values from imputation, respectively. Thus, 4 different full Bayesian LASSO analyses were carried out, each with 1,446 phenotypes and 42,552 regression coefficients but with varying proportions of actual and imputed genotypes. The resulting 42,552 estimated regression coefficients were then multiplied by corresponding genotype dosage values and summed across markers for 316 sires in the testing set. As before, genotypic data of sires in the testing set included $2,942,1,468,741$, or 366 actual genotypes and $39,610,41,084,41,811$, or 42,186 imputed genotype dosages, respectively, for masking rates of 93.1, 96.6, 98.3, or 99.1\%. Finally, the resulting DGV were correlated with April 2009 PTA from progeny testing for 316 Jersey sires in the testing set.

\section{RESULTS AND DISCUSSION}

Figure 1 shows the mean proportion of masked SNP genotypes that were imputed correctly (i.e., where the most likely genotype provided by IMPUTE 2.0 matched the original BovineSNP50 genotype call), averaged across animals, for 316 Jersey sires in the testing set. With a masking rate of $93.1 \%$, the proportion of genotypes that were imputed correctly ranged from 0.882 to 0.934 , with an average across chromosomes of 0.912 . With masking rates of $96.6,98.3$, or $99.1 \%$, corresponding means were $0.875,0.789$, or 0.735 , respectively, and ranges were 0.843 to $0.905,0.737$ to 0.836 , and 0.700 to 0.797 , respectively. These results are consistent with the imputation accuracies reported by Weigel et al. (2010), who showed in a population of 3,146 North American Jersey cattle that fastPHASE 1.2 (Scheet and Stephens, 2006) could provide accuracies of $0.869,0.776,0.709$, or 0.687, and IMPUTE 2.0 (Howie et al., 2009) could provide accuracies of $0.926,0.887,0.758$, or 0.712 when 90 , 95,98 , or $99 \%$ of BovineSNP50 genotypes were masked, respectively. Figure 1 also shows the proportion of masked SNP genotypes that were imputed correctly when masking was applied to 316 sires in the testing set plus 723 sires in the training set (i.e., a randomly chosen $50 \%$ ). In other words, the size of the reference panel from which haplotypes were estimated decreased from 1,446 to 723 , and the size of the study sample in which genotypes were imputed increased from 316 to 1,039. In this case, the mean proportions of genotypes that were imputed correctly were $0.896,0.860,0.782$, or 0.733 for masking rates of $93.1,96.6,98.3$, or $99.1 \%$, respectively. These proportions were $0.016,0.015,0.007$, or 0.002 lower, respectively, than when genotypes were masked only for sires in the testing set, indicating that halving the size of the reference panel led to a small reduction in imputation accuracy. The ranges in accuracy across chromosomes were 0.861 to $0.916,0.822$ to $0.893,0.727$ to 0.824 , or 0.695 to 0.783 , respectively, which were 


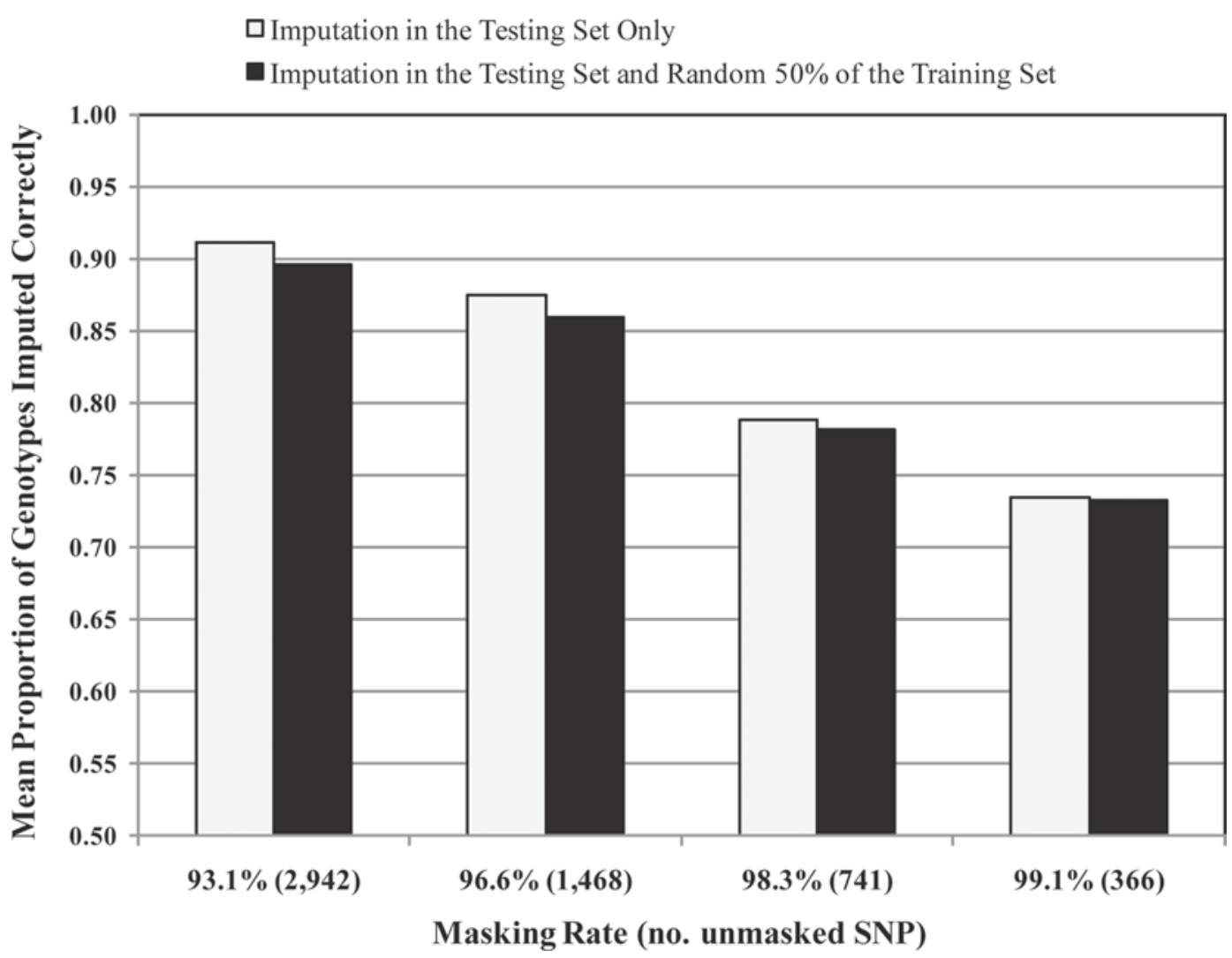

Figure 1. Mean proportion of masked SNP genotypes that were imputed correctly when genotypes were masked and subsequently imputed for bulls in the testing set only, and when genotypes were masked and subsequently imputed for bulls in the testing set plus a randomly chosen $50 \%$ of bulls in the training set.

only slightly greater than the ranges observed when the reference panel included all 1,446 sires.

Regardless of whether imputation was done in the testing set only or in the testing set and half of the training set, accuracy was reasonably high at masking rates of $93.1 \%$ and, to a lesser extent, $96.6 \%$. This seems to indicate that a low-density chip comprising 1,500 to 3,000 equally spaced SNP could provide sufficiently accurate imputation for applications such as preliminary screening of selection candidates or initial screening of chromosomal regions affecting a quantitative trait in a genome-wide association study. In addition to the number and spacing of SNP on a low-density platform, two other key considerations may affect the accuracy of imputation in practice. First is the extent of LD within a given breed (noting that LD phase is not conserved between breeds at the marker density provided by the BovineSNP50 BeadChip). The present study used data from North American Jersey cattle, which may have slightly greater LD than other common dairy cattle breeds. Recently, Villa-Angulo et al. (2009) reported that average $\mathrm{r}^{2}$ across 101 high-density regions of BTA6, BTA14, and BTA25 that were $100 \mathrm{~kb}$ in length, with an average of 19.6 SNP per region, was 0.380 in Jersey cattle and $0.377,0.333,0.323$, and 0.324 , respectively, in the Brown Swiss, Guernsey, Holstein, and Norwegian Red breeds. Our preliminary results (unpublished) suggest that imputation accuracy based on roughly 3,000 equally spaced SNP will be 3 to $4 \%$ lower in the Holstein breed compared with the Jersey breed. Second is the similarity of LD patterns between animals in the study sample, whose missing genotypes will be imputed, and their counterparts in the reference panel (typically ancestors), whose haplotypes will serve as the basis for imputation. Studies involving different ethnic groups in humans (e.g., Hao et al., 2009) have shown clearly that the individuals who make up the reference panel must be representative of the genetic background of individuals in the study sample. Although this might seem obvious, situations may arise in practice in which some animals in the study sample result from crossbreeding, importation of genetic material from another country, or selection for atypical traits (e.g., polled condition or red coat color in Holsteins); in such situations, the accuracy of imputed genotypes may be poorer than anticipated. 
Table 3. Posterior means of the residual variance $\left(\sigma_{\varepsilon}^{2}\right)$ and the regularization parameter $(\lambda)$ for milk yield, protein percentage, and daughter pregnancy rate (DPR) when full or reduced Bayesian least absolute selection and shrinkage operator (LASSO) models with varying numbers of SNP covariates were fitted to data from the training set ${ }^{1}$

\begin{tabular}{lccccc}
\hline & \multicolumn{5}{c}{ SNP covariates in Bayesian LASSO model, n } \\
\cline { 2 - 5 } Item & 42,552 & 2,942 & 1,468 & 741 & 366 \\
\hline Residual variance $\left(\sigma_{\varepsilon}^{2}\right)$ & & & & \\
Milk yield & 0.098 & 0.162 & 0.247 & 0.321 & 0.417 \\
Protein percentage & 0.081 & 0.184 & 0.313 & 0.452 & 0.556 \\
DPR & 0.171 & 0.280 & 0.356 & 0.431 & 0.533 \\
Regularization parameter $(\lambda)$ & 67.6 & 27.3 & 25.0 & 21.3 & 19.2 \\
Milk yield & 51.6 & 24.6 & 24.4 & 24.2 & 22.3 \\
Protein percentage & 82.3 & 35.5 & 30.2 & 25.6 & 22.2 \\
DPR & & & & \\
\hline
\end{tabular}

${ }^{1}$ Note that phenotypes for each trait were standardized to have a sample variance equal to 1.

Table 3 shows the posterior means of the residual variance $\left(\sigma_{\varepsilon}^{2}\right)$ from the Bayesian LASSO model. Given that PTA were standardized to have a sample variance equal to 1 , the residual variance can be interpreted as the proportion of variance that was not explained by the model, noting that a small residual variance might also reflect over-fitting of data from the training set in models with an extremely large number of explanatory variables. In the reference model, in which no genotypes were masked and 42,552 SNP covariates were used for estimation of SNP effects, estimates of the residual variance were $0.098,0.081$, and 0.171 , respectively, for milk yield, protein percentage, and DPR. This indicates slightly greater model fit for protein percentage than for milk yield, but substantially poorer fit for DPR than for either of the production traits. As the number of SNP covariates in the reduced Bayesian LASSO models (which, in this case, contained only SNP that remained unmasked in the testing set) decreased from 2,942 to 366 , the residual variances for milk yield, protein percentage, and DPR increased from 0.162 to $0.417,0.184$ to 0.556 , and 0.280 to 0.533 , respectively. As expected, goodness of fit decreased as the number of covariates in the model decreased. Also, these results showed that goodness of fit was consistently greater for milk yield than for protein percentage or DPR.

Table 4 shows estimates of the residual variance obtained when models were fitted to a training set in which $50 \%$ of the sires had imputed genotypes at varying masking rates (93.1 to $99.1 \%$ ). For a masking rate of $93.1 \%$, the estimated residual variances for milk yield, protein percentage, and DPR increased by 31,54 , and $37 \%$, respectively, relative to the reference model (see Table 3). As the proportion of masked SNP increased from 96.6 to $99.1 \%$, estimated residual variances increased by 65,143 , and $242 \%$ for milk yield; 111,243 , and $374 \%$ for protein percentage; and 46,87 , and $154 \%$ for DPR, respectively, relative to the reference model. It is important to note that, in every case, the estimated residual variances from models in which the majority of the 42,552 SNP covariates were imputed in half of the sires in the training set were substantially smaller than estimates from corresponding reduced models in which only $2,942,1,468,741$, or 366 unmasked SNP genotypes were used as covariates. For example, at a masking rate of $93.1 \%$, estimated residual variances from the models with 42,552 SNP covariates (imputed for half of the sires in the training set) were 21,32 , and $16 \%$ lower for milk yield, protein percentage, and DPR, respectively, than estimates from the corresponding reduced model with 2,942 unmasked SNP covariates (see Table 3). As noted earlier, a smaller estimated residual variance indicates better fit of the model to data from the training set, but this does not guarantee greater predictive ability when this model is applied to data from an independent testing set.

Tables 3 and 4 also show estimates of the regularization parameter $(\lambda)$ for the shape of the prior distribution of the scaling parameter $\left(\tau_{j}^{2}\right)$ controlling the prior precision variance of the $j$ th marker effect. As $\lambda$ approaches zero, the Bayesian LASSO model tends toward ordinary least squares, whereas large values of $\lambda$ increase the extent of shrinkage of estimates of marker effects. In the present study, estimates of $\lambda$ were 67.6 , 51.6 , and 82.3 , respectively, for the reference model with 42,552 SNP covariates and no masking (see Table 3). In reduced Bayesian LASSO models with 2,942 to 366 SNP covariates, estimates of $\lambda$ decreased from 27.3 to 19.2 for milk yield, 24.6 to 22.3 for protein percentage, and 35.5 to 22.2 for DPR (see Table 3). Estimates of $\lambda$ from models with 42,552 SNP covariates, in which 93.1 to $99.1 \%$ of genotypes were imputed for half of the 
Table 4. Posterior means of the residual variance $\left(\sigma_{\varepsilon}^{2}\right)$ and the regularization parameter $(\lambda)$ for milk yield, protein percentage, and daughter pregnancy rate (DPR) when full Bayesian least absolute selection and shrinkage operator (LASSO) models were fitted to data from a training when $50 \%$ of the sires had imputed genotypes at different masking rates ${ }^{1}$

\begin{tabular}{lcccc}
\hline & \multicolumn{4}{c}{ Masking rate, \% (number of unmasked SNP genotypes) } \\
\cline { 2 - 5 } Item & $93.1(2,942)$ & $96.6(1,468)$ & $98.3(741)$ & $99.1(366)$ \\
\hline Residual variance $\left(\sigma_{\varepsilon}^{2}\right)$ & & & & \\
Milk yield & 0.128 & 0.162 & 0.238 & 0.335 \\
Protein percentage & 0.125 & 0.171 & 0.278 & 0.384 \\
DPR & 0.234 & 0.250 & 0.319 & 0.435 \\
Regularization parameter $(\lambda)$ & 73.6 & 82.2 & 99.7 & 112.3 \\
Milk yield & 61.1 & 71.5 & 94.2 & 109.6 \\
Protein percentage & 97.8 & 99.9 & 112.2 & 131.2 \\
DPR & & & & \\
\hline
\end{tabular}

${ }^{1}$ Note that phenotypes for each trait were standardized to have a sample variance equal to 1 .

sires in the training set, ranged from 73.6 to 112.3 for milk yield, 61.1 to 109.6 for protein percentage, and 97.8 to 131.2 for DPR, and $\lambda$ consistently increased as the masking rate increased; that is, as the proportion of imputed SNP increased (see Table 4). In all models that were based on imputed genotypes, estimates of $\lambda$ were greater than estimates from the reference model. This indicates that the distribution of estimated SNP effects tended to have greater mass around zero (i.e., thinner tails) when the majority of SNP genotypes were imputed for half of the sires in the training set, and the proportion of mass at zero increased as the proportion of imputed genotypes increased.

Figures 2, 3, and 4 show the correlations between the DGV obtained by applying estimated SNP effects derived from May 2006 data from sires in the training set to actual or imputed genotypes of sires in the testing set and the April 2009 progeny test PTA of sires in the testing set for milk yield, protein percentage, and DPR, respectively. Again, values denoted as reference correspond to the full Bayesian LASSO model in which none of the 42,552 SNP genotypes were masked in the training set or testing set. For a masking rate of $93.1 \%$, reduced models with 2,942 SNP covariates for milk yield, protein percentage, and DPR provided correlations of $0.617,0.687$, and 0.608 , respectively, compared with reference correlations of $0.673,0.770$, and 0.674 , respectively. When SNP genotypes were imputed for sires in the testing set only and 42,552 SNP covariates were used in the Bayesian LASSO model, correlations were $0.673,0.740$, and 0.642 , respectively. Likewise, when SNP genotypes were imputed for sires in the testing set and half of the sires in the training set, corresponding correlations were $0.640,0.690$, and 0.641 , respectively. Averaged across traits at a masking rate of $93 \%$, predictive ability was approximately $97 \%$ as great as that of the reference model when genotypes were imputed in the testing set only, and about $93 \%$ as great when genotypes were imputed in the testing set and half of the training set. In most applications of genomic selection in dairy cattle, the testing set consists largely of animals that are progeny or grandprogeny of animals in the training set. Thus, the approach suggested by Habier et al. (2009), namely re-genotyping of the selected parents in each generation with a high-density assay, should be an effective strategy to maximize the accuracy of DGV of young selection candidates. It is important to recognize that high-density genotypes of both parents were required in the simulation study of Habier et al. (2009), and therefore relatively few equally spaced SNP were required to generate accurate DGV of the progeny. In the present study, only a reference population consisting of representative animals of the same breed is needed. In practice, high-density genotypes will be unavailable for many of the dams of animals that are genotyped with low-density assays on commercial farms.

When $96.6 \%$ of SNP were masked in the testing set only, the correlations between DGV and progeny test PTA were $0.649,0.676$, and 0.619 , respectively, for milk yield, protein percentage, and DPR using full models with 42,552 (mostly) imputed SNP genotypes, compared with $0.515,0.614$, and 0.585 for reduced models with 1,468 actual SNP genotypes. When genotypes were also imputed for half of the sires in the training set, correlations decreased to $0.628,0.658$, and 0.610 , respectively. Thus, correlations obtained with imputation were clearly superior to those obtained by deleting these loci from the analysis.

With a masking rate of $98.3 \%$, correlations between DGV and progeny-test PTA in the testing set were 0.525 , 0.546 , and 0.572 for milk yield, protein percentage, and DPR, respectively, when genotypes were imputed in the testing set only. By comparison, correlations were 


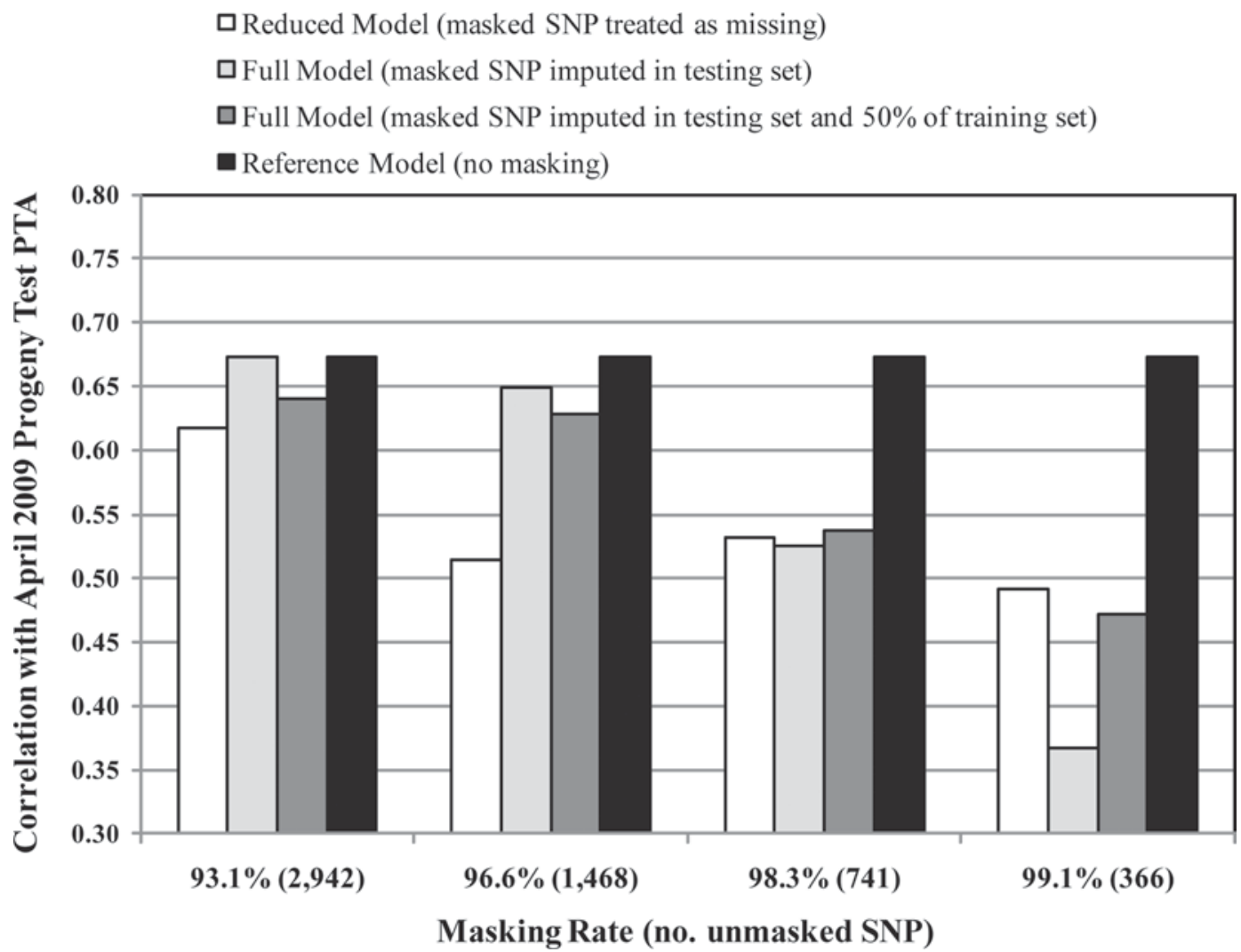

Figure 2. Correlations between predicted direct genomic values for milk yield and corresponding April 2009 progeny-test PTA using full or reduced models with 42,552 or $366,741,1,468$, or 2,942 single SNP covariates, respectively, with or without imputation of masked genotypes for bulls in the testing set or bulls in the testing set and a randomly chosen $50 \%$ of bulls in the training set. The bars denoted as "reference" correspond to correlations from a full model in which all 42,552 SNP genotypes were left as unmasked in both the training and testing sets.

$0.537,0.534$, and 0.546 , respectively, when genotypes were imputed in the testing set and half of the training set, and $0.532,0.539$, and 0.544 , respectively, when reduced models containing 741 unmasked SNP were applied. Last, with a masking rate of $99.1 \%$, imputation of masked genotypes in the testing set provided correlations of only $0.367,0.468$, and 0.470 for milk yield, protein percentage, and DPR, respectively, compared with $0.472,0.506$, and 0.506 , respectively, for imputation in the testing set and half of the training set, and $0.492,0.504$, and 0.518 , respectively, for reduced models containing only 366 unmasked SNP. At a masking rate of $99.1 \%$, predictive ability tended to be greater for reduced models in which loci with missing genotypes had been deleted, rather than full models in which missing genotypes had been imputed, presumably because of poor imputation accuracy. Paradoxically, for a masking rate of $99.1 \%$, correlations were higher when genotypes were imputed in the testing set and half of the training set, compared with imputation in the testing set only, despite the fact that the reference panel for imputation was significantly smaller (723 sires vs.1,446 sires) and the accuracy of imputation was slightly lower (0.733 vs. 0.735). A likely explanation is that because many sires in the testing set were close relatives (e.g., progeny) of the random $50 \%$ of sires in the training set that were subject to masking, some of the haplotyping errors may have been consistent between the training and testing sets. In such a case, imputation errors would not occur randomly, but rather they would be consistent within families and could serve as a proxy for pedigree relationships, and this would lead to an unexpected gain in predictive ability. Nonetheless, predictive ability tended to be poor for all models at masking rates of 98.3 and $99.1 \%$, and neither imputation of missing SNP genotypes nor application of reduced models with actual genotypes for equally spaced, low-density SNP is recommended.

Recently, Vazquez et al. (2009) showed that correlations between DGV and progeny test PTA of Holstein sires for milk yield using $1,500,750$, or 375 selected SNP with largest estimated effects on milk yield were approximately 94,88 , or $84 \%$ as great, respectively, as those provided by the full set of 32,518 SNP. Corre- 


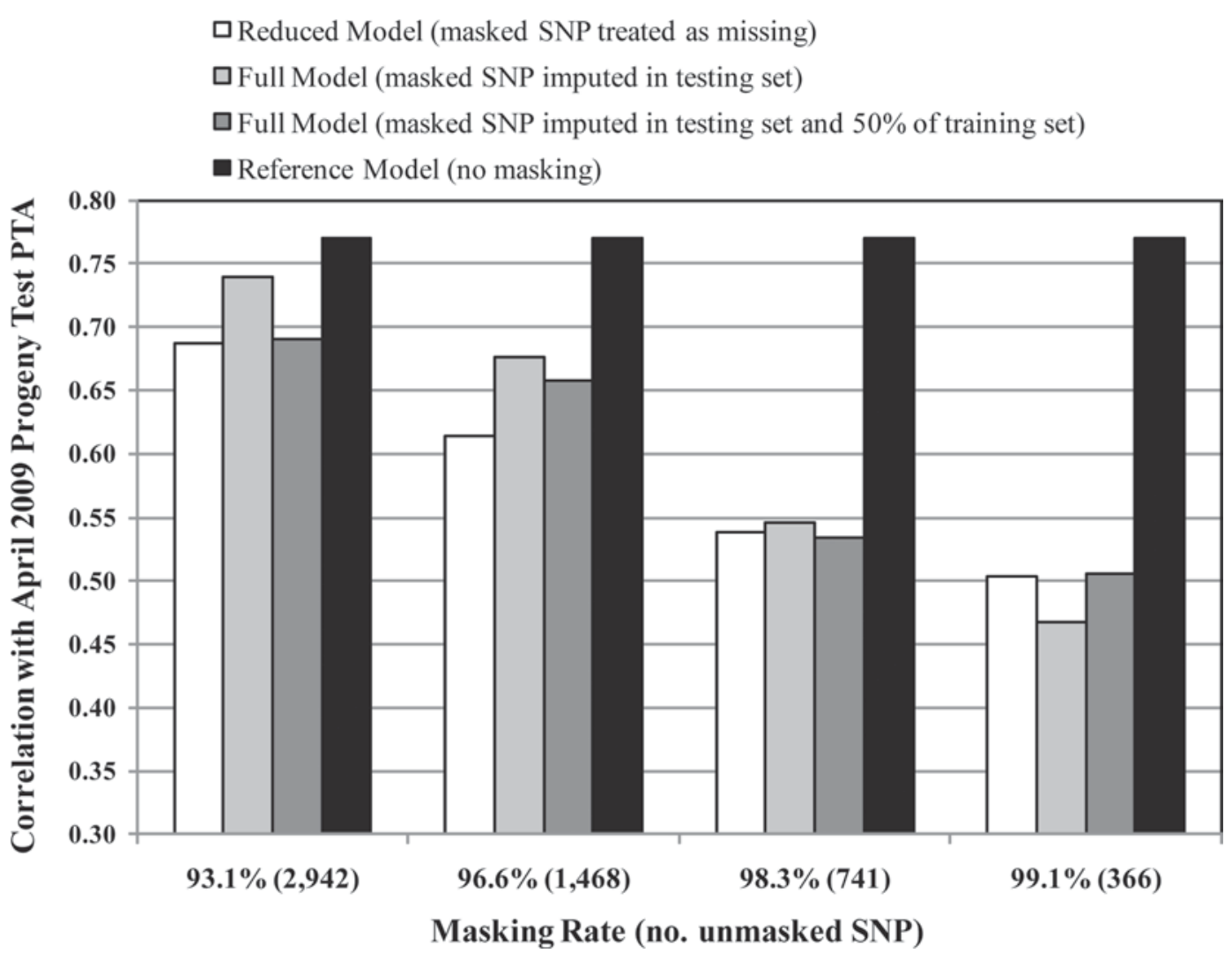

Figure 3. Correlations between predicted direct genomic values for protein percentage and corresponding April 2009 progeny-test PTA using full or reduced models with 42,552 or $366,741,1,468$, or 2,942 SNP covariates, respectively, with or without imputation of masked genotypes for bulls in the testing set or bulls in the testing set and a randomly chosen $50 \%$ of bulls in the training set. The bars denoted as "reference" correspond to correlations from a full model in which all 42,552 SNP genotypes were left as unmasked in both the training and testing sets.

sponding ratios for DPR were approximately 89, 85, or $78 \%$, respectively. However, assays developed using SNP that were selected for individual traits such as milk yield or DPR would, by definition, be trait-specific. Correlations between DGV and progeny-test PTA for milk yield using 1,500, 750 , or 375 selected SNP with largest effects on lifetime net merit were roughly 79 , 76 , or $70 \%$ as great, respectively, as those provided by the full SNP set, whereas corresponding ratios for DPR were 80,74 , or $63 \%$, respectively. In comparison, ratios obtained in the present study using $2,942,1,468,741$, or 366 equally spaced SNP with imputation were 97 , 92,78 , or $62 \%$, respectively, averaged across the traits of milk yield, protein percentage, and DPR. Thus, it appears that genotyping 1,500 to 3,000 equally spaced SNP with subsequent imputation of high-density genotypes would provide greater predictive ability than genotyping a comparable number of selected SNP with large estimated effects. On the other hand, if cost considerations limit genotyping to 750 loci or fewer, assays based on selected SNP with large estimated effects on lifetime net merit may be preferred.
Many recent studies involving humans and other model or food animal species have focused on construction of haplotypes (e.g., Khatkar et al., 2007; Kong et al., 2008), which can be used directly when carrying out genome-wide association studies involving a particular region of the genome (e.g., Druet and Georges, 2010) or making inferences about the evolutionary history or substructure of a given population (e.g., Lohmueller et al., 2009). In other cases, haplotypes may simply represent an intermediate step in the process of imputing missing genotypes or computing genotype probabilities, as was the case in the present study using the IMPUTE2.0 software. Algorithms that utilize both pedigree and genomic information (e.g., Albers et al., 2007; Ding et al., 2007) might provide enhanced imputation accuracy, given that extensive pedigree recording has occurred in most livestock populations during the past century. For this reason, a major effort is underway to prepare national genetic evaluation systems for the arrival of data from low-density and ultra-high-density genotyping platforms. Specifically, the challenge is to integrate new genomic information into the extensive 


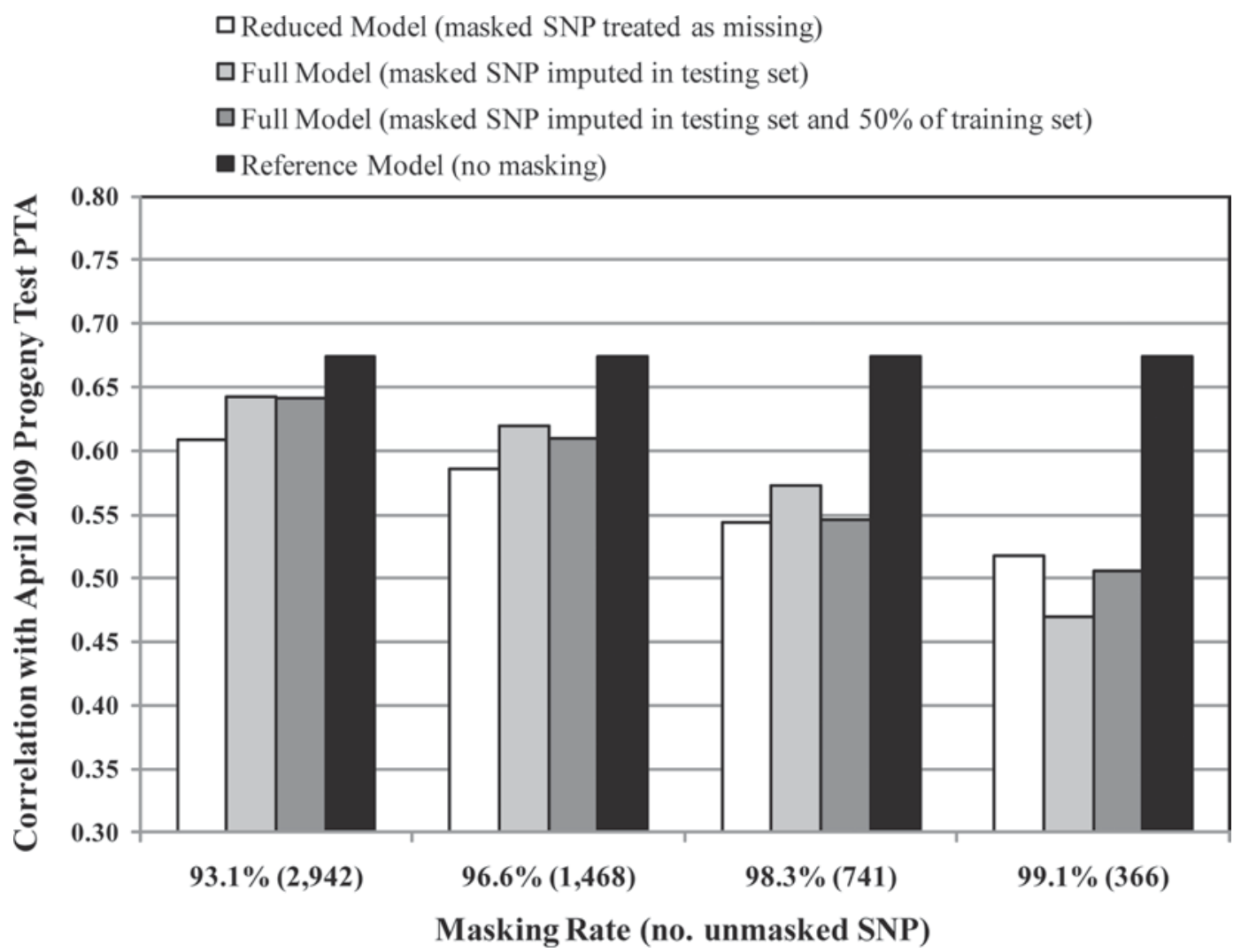

Figure 4. Correlations between predicted direct genomic values for daughter pregnancy rate and corresponding April 2009 progeny-test PTA using full or reduced models with 42,552 or $366,741,1,468$, or 2,942 SNP covariates, respectively, with or without imputation of masked genotypes for bulls in the testing set or bulls in the testing set and a randomly chosen $50 \%$ of bulls in the training set. The bars denoted as "reference" correspond to correlations from a full model in which all 42,552 SNP genotypes were left as unmasked in both the training and testing sets.

genotypic databases that have been compiled using the BovineSNP50 BeadChip, and a likely solution involves construction of haplotypes and subsequent imputation of genotypes using both genomic and pedigree information (G. R. Wiggans, Animal Improvement Programs Laboratory, ARS, USDA, Beltsville, MD; personal communication).

\section{CONCLUSIONS}

Results of the study described herein indicate that low-density genotyping of selection candidates using a panel of roughly 3,000 equally spaced SNP, followed by imputation of missing high-density genotypes from a reference panel of 1,500 or more animals with similar genetic background, can provide approximately $95 \%$ of the predictive ability achieved through high-density genotyping of all selection candidates. Accuracy of imputation, and hence predictive ability, may be slightly greater for Jersey cattle than for other common dairy breeds because of the smaller effective population size. Regardless, the level of accuracy should be sufficient for commercial applications such as prescreening of young bulls that are candidates for AI programs, screening of lactating cows on commercial dairies that may offer superior or unique genetic contributions, or selection among extra replacement heifers that have been generated by using sex-sorted semen. If such genotyping platforms can be offered at a modest price, the technology of genomics may extend its reach beyond AI companies and pedigree breeders to achieve routine application on commercial dairy farms. In the future, the accuracy of breeding values derived from low-density genotypes may be compromised if high-density genotypes are lacking for a significant proportion of animals in the training set, from which SNP effects are estimated, so re-genotyping of selected individuals (e.g., males to be marketed through AI programs) will likely be justified.

\section{ACKNOWLEDGMENTS}

This project was supported by National Research Initiative competitive grant no. 2009-35205-05099 from 
the USDA National Institute for Food and Agriculture Animal Genome Program (Washington, DC). Data for this study were provided by the USDA-ARS Animal Improvement Programs Laboratory (Beltsville, MD) and the American Jersey Cattle Association (Reynoldsburg, $\mathrm{OH}$ ). The IMPUTE 2.0 software was provided by Bryan Howie and Jonathan Marchini, Department of Statistics, University of Oxford (United Kingdom). Kent Weigel acknowledges financial support from the National Association of Animal Breeders (Columbia, $\mathrm{MO})$.

\section{REFERENCES}

Albers, C. A., T. Heskes, and H. J. Kappen. 2007. Haplotype inference in general pedigrees using the cluster variation method. Genetics 177:1101-1116.

de los Campos, G., H. Naya, D. Gianola, J. Crossa, A. Legarra, E. Manfredi, K. Weigel, and J. M. Cotes. 2009. Predicting quantitative traits with regression models for dense molecular markers and pedigree. Genetics 182:375-385.

Ding, X. D., H. Simianer, and Q. Zhang. 2007. A new method for haplotype inference including full-sib information. Genetics 177:1929-1940.

Druet, T., and M. Georges. 2010. A hidden Markov model for combining linkage and linkage disequilibrium information for haplotype reconstruction and QTL fine mapping. Genetics 184:789-798. doi:10.1534/genetics.109.108431

Habier, D., R. L. Fernando, and J. C. M. Dekkers. 2009. Genomic selection using low-density marker panels. Genetics 182:343-353.

Halperin, E., and D. Stephan. 2009. SNP imputation in association studies. Nat. Biotechnol. 27:349-351.

Hao, K., E. Chudin, J. McElwee, and E. Schadt. 2009. Accuracy of genome-wide imputation of untyped markers and impacts on statistical power for association studies. BMC Genet. doi:10.1186/14712156/10-27

Hayes, B. J., P. J. Bowman, A. J. Chamberlain, and M. E. Goddard. 2009. Invited review: Genomic selection in dairy cattle: Progress and challenges. J. Dairy Sci. 92:433-443.

Howie, B. N., P. Donnelly, and J. Marchini. 2009. A flexible and accurate genotype imputation method for the next generation of genome-wide association studies. PLoS Genet. 5:e1000529.

Khatkar, M. S., K. R. Zenger, M. Hobbs, R. J. Hawken, J. A. L. Cavanagh, W. Barris, A. E. McClintock, S. McClintock, P. C. Thomson, B. Tier, F. W. Nicholas, and H. W. Raadsma. 2007. A primary assembly of a bovine haplotype block map based on a 15,036-single-nucleotide polymorphism panel genotyped in Holstein-Friesian cattle. Genetics 176:763-772.

Kong, A., G. Masson, M. L. Frigge, A. Gylfason, P. Zusmanovich, G. Thorleifsson, P. I. Olason, A. Ingason, S. Steinberg, T. Rafnar, P. Sulem, M. Mouy, F. Jonsson, U. Thorsteinsdottir, D. F. Gud- bjartsson, H. Stefansson, and K. Stefansson. 2008. Detection of sharing by descent, long-range phasing and haplotype imputation. Nat. Genet. 40:1068-1075.

Lohmueller, K. E., C. D. Bustamante, and A. G. Clark. 2009. Methods for human demographic inference using haplotype patterns from genomewide single-nucleotide polymorphism data. Genetics 182:217-231.

Meuwissen, T. H. E., B. J. Hayes, and M. E. Goddard. 2001. Prediction of total genetic value using genome-wide dense marker maps. Genetics 157:1819-1829.

Park, T., and G. Casella. 2008. The Bayesian Lasso. J. Am. Stat. Assoc. 103:681-686.

R Development Core Team. 2009. R: A language and environment for statistical computing. http://www.R-project.org. R Foundation for Statistical Computing, Vienna, Austria.

Scheet, P., and M. Stephens. 2006. A fast and flexible statistical model for large-scale population genotype data: Applications to inferring missing genotypes and haplotypic phase. Am. J. Hum. Genet. 78:629-644.

Van Tassell, C. P., T. P. L. Smith, L. K. Matukumalli, J. F. Taylor, R. D. Schnabel, C. T. Lawley, C. D. Haudenschild, S. S. Moore, W. C. Warren, and T. S. Sonstegard. 2008. SNP discovery and allele frequency estimation by deep sequencing of reduced representation libraries. Nat. Methods 5:247-252.

VanRaden, P. M., C. P. Van Tassell, G. R. Wiggans, T. S. Sonstegard, R. D. Schnabel, J. F. Taylor, and F. Schenkel. 2009. Reliability of genomic predictions for North American dairy bulls. J. Dairy Sci. $92: 16-24$.

Vazquez, A. I., G. J. M. Rosa, K. A. Weigel, G. de los Campos, and D. Gianola. 2009. Selection of SNPs for an optimal low-density assay for prediction of genetic values. J. Dairy Sci. 92(Suppl. 1):125. (Abstr.)

Villa-Angulo, R., L. K. Matukumalli, C. A. Gill, J. Choi, C. P. Van Tassell, and J. J. Grefenstette. 2009. High-resolution haplotype block structure in the cattle genome. BMC Genet. 10:19.

Weigel, K. A., G. de los Campos, O. González-Recio, H. Naya, X. L. Wu, N. Long, G. J. M. Rosa, and D. Gianola. 2009. Predictive ability of direct genomic values for lifetime net merit of Holstein sires using selected subsets of single nucleotide polymorphism markers. J. Dairy Sci. 92:5248-5257.

Weigel, K. A., C. P. Van Tassell, J. R. O'Connell, P. M. VanRaden, and G. R. Wiggans. 2010. Prediction of unobserved single nucleotide polymorphism genotypes of Jersey cattle using reference panels and population-based imputation algorithms. J. Dairy Sci. 93:2229-2238.

Wiggans, G. R., P. M. VanRaden, L. R. Bacheller, F. A. Ross Jr, T. S. Sonstegard, G. te Meerman, and C. P. Van Tassell. 2009. Transition of genomic evaluation from a research project to a production system. J. Dairy Sci. 92(E-Suppl. 1):313-314. (Abstr.)

Zimin, A. V., A. L. Delcher, L. Florea, D. R. Kelley, M. C. Schatz, D. Puiu, F. Hanrahan, G. Pertea, C. P. Van Tassel, T. S. Sonstegard, G. Marçais, M. Roberts, P. Subramanian, J. A. Yorke, and S. L. Salzberg. 2009. A whole-genome assembly of the domestic cow, Bos taurus. Genome Biol. 10:R42. 